\title{
The Second Orthogonality Conditions in the Theory of Proper and Improper Rotations IV. Solution of the Trace and Secular Equations*
}

\author{
Harry Gelman**
}

(February 25, 1969)

\begin{abstract}
The equation which connects the trace of a rotation matrix and that of its square, and the secular equation for a rotation matrix, both of which are direct results of the second orthogonality conditions, are solved by purely analytic methods based on the group property and the periodicity property of rotation matrices. The point is thus made that the well known formulas for the trace of a rotation matrix in terms of the angle of rotation and for the rotation matrix itself in terms of the axis and angle of rotation, are closely related to the algebraic properties of rotation matrices.
\end{abstract}

Key words: Closed form of rotation matrix; orthogonal transformation; rotation; rotation matrix; secular equation; trace equation; trace formula.

\section{Introduction}

In paper I $[1]^{1}$ of this series two of the results derived from the second orthogonality conditions were an equation which connects the trace of an orthogonal transformation matrix with that of its square, and the secular equation for a transformation matrix. It was stated that for rigid rotations these equations could be solved directly by analytic methods based only on very general properties of rotation matrices. The details of the solutions were deferred to the present paper.

For completeness we rewrite here the equations to be solved in this paper and the solutions, using $R$ to denote a rotation matrix. The equation which connects $\operatorname{tr} R$ and $\operatorname{tr}\left(R^{2}\right)$ is eq (I 15) with $p^{\prime} / p=1$,

$$
(\operatorname{tr} R)^{2}-\operatorname{tr}\left(R^{2}\right)=2 \operatorname{tr} R .
$$

The secular equation for $R$ is eq (I 22) with $p^{\prime} / p=1$,

$$
R^{3}-(\operatorname{tr} R) R^{2}+(\operatorname{tr} R) R-I=0,
$$

where $I$ is the identity matrix. The well known expression for $\operatorname{tr} R$ in terms of the rotation angle $\alpha$ is

$$
\operatorname{tr} R=1+2 \cos \alpha \text {. }
$$

When this is inserted in eq (2), the secular equation becomes

$$
R^{3}-(1+2 \cos \alpha) R^{2}+(1+2 \cos \alpha) R-I=0 .
$$

The solution of eq (4) is

$$
R(\alpha)=I-N \sin \alpha+N^{2}(1-\cos \alpha)
$$

*An invited paper. This work was supported by the U.S. Air Force, Electronic Systems Division, Air Force Systems Command under contract No. AF19(628)5165, Project 4966.

**Present address: The MITRE Corporation, Bedford, Mass. 01730.

${ }^{1}$ Figures in brackets indicate the literature references at the end of this paper. 
where $N$ is given in terms of the components of the axis of rotation by eqs (I 26) and (I 27). The end results of this paper are derivations of eq (3) from eq (1) and eq (5) from eq (4).

The properties of rotation matrices which the derivations in this paper are based on are the group property and the periodicity property. The group property follows from the fact that the product of two rotation matrices is a matrix which has determinant unity and satisfies the first orthogonality conditions, and therefore represents a rotation about some axis. The computation of the axis and angle of the product rotation was treated as an example in section 4 of paper II [2]. For the purposes of this paper a restricted statement of the group property suffices. This is the statement that the product of two matrices representing rotations about the same axis is a matrix representing a rotation about that axis by an angle which is the sum of the angles in the component rotations. If $R(\alpha)$ represents a rotation about a fixed axis by an angle $\alpha$, the restricted statement of the group property is

$$
R\left(\alpha_{2}\right) R\left(\alpha_{1}\right)=R\left(\alpha_{1}+\alpha_{2}\right) .
$$

The second property of rotation matrices that we use is the periodicity property. We take as the statement of this property the equality of $R(2 \pi)$ (for any axis) with $R(0)$, which is the identity matrix,

$$
R(2 \pi)=R(0)=I,
$$

together with the requirement that for no angle smaller than $2 \pi$ is $R(\alpha)$ equal to the identity. The second part of the statement is necessary in order that $\alpha$ be the true angle of rotation rather than some multiple of it. From eqs (6) and (7) an alternative form of the periodicity property is

$$
R(\alpha+2 \pi)=R(\alpha) R(2 \pi)=R(\alpha) .
$$

The solutions eqs (3) and (5) are well known and are not the objects of interest in this paper. The objects of interest are the derivations of these solutions to be presented in sections 2 and 3 respectively. The aim in giving these new derivations is to call attention to the fact that the trace formula eq (3) and the closed form eq (5) are direct consequences of algebraic properties of rotation matrices. Thus, although the derivations given here do not give much geometrical insight into the nature of the solutions, they may give a deeper insight.

\section{Solution of the Trace Equation}

We represent the dependence ${ }^{2}$ of $\operatorname{tr} R$ on $\alpha$ by the function $g(\alpha)$,

$$
g(\alpha) \equiv \operatorname{trR}(\alpha) \cdot
$$

We now observe from eq (6) that $[R(\alpha)]^{2}=R(2 \alpha)$. It then follows from eq (9) that

$$
\operatorname{tr}[R(\alpha)]^{2}=\operatorname{tr} R(2 \alpha)=g(2 \alpha) \cdot
$$

We may therefore rewrite eq (1) as

$$
[g(\alpha)]^{2}-g(2 \alpha)=2 g(\alpha),
$$

which is a functional equation for the unknown function $g(\alpha)$. A more transparent functional equation is obtained by defining the function

$$
h(\alpha) \equiv \frac{1}{2}[g(\alpha)-1],
$$

and rewriting eq (11) in terms of $h$. The functional equation for $h$ can be written

$$
[h(\alpha)]^{2}=\frac{1}{2}[h(2 \alpha)+1] .
$$

Equations (11) and (13) are both independent of the axis of rotation.

\footnotetext{
${ }^{2}$ The fact that $g(\alpha)$ is independent of the axis of rotation is not immediately apparent from eq (1). It will become apparent as the derivation proceeds.
} 
From eq (13) one can begin to see the connection between the result $h(\alpha)=\cos \alpha$, and eq (1). The structure of eq (13) is identical to that of the formula for the cosine of a double angle. However, $\cos \alpha$ is not the only solution to eq (13). We will calculate all of its solutions and then see how the geometrically meaningful solution is selected by the periodicity condition.

The coordinate transformations which underlie rotation matrices are analytic functions of the parameters which specify a rotation. ${ }^{3}$ It follows from this that $h(\alpha)$ can be expanded in a convergent real Taylor series about $\alpha=0$. Now we observe from eq (13) that if $h(\alpha)$ is a solution, so also is. $h(-\alpha)$. That is, $h(\alpha)$ is an even function of $\alpha$, and when expanded in a power series about $\alpha=0$ contains only even powers of $\alpha$. We therefore write the assumed series in the form

$$
h(\alpha)=\sum_{m=0}^{\infty} C_{2 m} \alpha^{2 m}
$$

where the $C_{j}$ are constant expansion coefficients. The square of this series is

$$
[h(\alpha)]^{2}=\sum_{m=0}^{\infty} \sum_{n=0}^{\infty} C_{2 m} C_{2 n} \alpha^{2(m+n)} .
$$

We notice that in $h^{2}, \alpha$ occurs raised to the power $2(m+n)$. This suggests that we change the variables of summation in eq (15) from $m$ and $n$, to a pair of variables one of which is $m+n$. Accordingly we define new variables $j$ and $k$ by $j \equiv m+n, k \equiv m-n$. To find the ranges of $j$ and $k$ we note that $j$ is nonnegative and increases in steps of one. Hence its range is all of the nonnegative integers. We next observe that for a given value of $j$, there are $j+1$ allowed pairs of values of $m$ and $n$ in which the sum of the values of $m$ and $n$ in each pair equals the given $j$ value. These pairs $(m, n)$ are $(0, j),(1, j-1),(2, j-2), \ldots,(j-1,1),(j, 0)$. The values of $k$ corresponding to these pairs are $k=-j,-j+2,-j+4, \ldots ., j-2, j$. We see that $k$ ranges from $-j$ to $+j$ in steps of two. It is convenient to parametirize $k$ as $k=-j+2 r$ where $r$ is an integer taking on the values $0,1,2, \ldots, j$. Since the subscripts on the $C$ 's in eq (15) are $2 m$ and $2 n$, we have to express these in terms of $j$ and $r$. The results are $2 m=2 r, 2 n=2(j-r)$. The series for $h^{2}$ may then be written

$$
[h(\alpha)]^{2}=\sum_{j=0}^{\infty}\left[\sum_{r=0}^{j} C_{2 r} C_{2(j-r)}\right] \alpha^{2 j} .
$$

Note that the order of summation in eq (16) cannot be interchanged since the range of $r$ depends upon $j$.

If we insert the series eq (14) evaluated for argument $2 \alpha$, and the series eq (16) for argument $\alpha$ into the functional equation (13), we get

$$
\sum_{j=0}^{\infty}\left[\sum_{r=0}^{j} C_{2 r} C_{2(j-r)}\right] \alpha^{2 j}=\frac{1}{2}\left[\sum_{j=0}^{\infty} 2^{2 j} C_{2 j} \alpha^{2 j}+1\right] .
$$

By equating the coefficients of like powers of $\alpha$ on the two sides of this equation we arrive at a set of equations for the determination of the $\mathrm{C}_{2 j}$. We break this process into the cases $j=0, j=1$, and $j \geqslant 2$.

(a) $j=0$. This yields an equation for $\mathrm{C}_{0}$,

$$
C_{0}^{2}=\frac{1}{2}\left(C_{0}+1\right),
$$

whose solutions are $C_{0}=-\frac{1}{2}$, and $C_{0}=1$. The former solution can be shown to generate a "series" all of whose higher order coefficients are zero. This corresponds to the constant solution $h(\alpha)=-\frac{1}{2}$ of eq (13), or by eq (12) to the trivial solution $g(\alpha)=0$ of eq (11). This solution can be ruled out because it has arbitrarily small periods in $\alpha$, whereas the second part of the periodicity condition requires that there be no period smaller than $2 \pi$. We therefore concentrate on the series generated by the value $C_{0}=1$.

(b) $j=1$. Equating coefficients of $\alpha^{2}$ on both sides of eq (17) we get

$$
C_{0} C_{2}=C_{2},
$$

${ }^{3}$ This is equivalent to saying that the rotation group is a Lie group. 
and setting $C_{0}=1$ we get the identity $C_{2}=C_{2}$. This means that $C_{2}$ is not determined by eq (13) and is therefore a free parameter of the solution. All of the suceeding coefficients will depend on the value of $C_{2}$.

(c) $j \geqslant 2$. Equating the coefficients of $\alpha^{2 j}$ on both sides of eq (17) we have

$$
\sum_{r=0}^{j} C_{2 r} C_{2(j-r)}=2^{2 j-1} C_{2 j}
$$

Now since $j \geqslant 2$ we can write the first and last terms (i.e., those for $r=0$ and $r=j$ ) on the left side of eq (20) separately from the rest of the sum, giving

$$
2 C_{0} C_{2 j}+\sum_{r=1}^{j-1} C_{2 r} C_{2(j-r)}=2^{2 j-1} C_{2 j}
$$

Upon setting $C_{0}=1$ this equation can be solved for $C_{2 j}$ to give

$$
C_{2 j}=\frac{1}{2\left[2^{2(j-1)}-1\right]} \sum_{r=1}^{j-1} C_{2 r} C_{2(j-r)}
$$

This is a recursion relation for $C_{2 j}$.

Computation of the first few coefficients from eq (22) gives

$$
\begin{aligned}
& C_{4}=\frac{C_{2}^{2}}{6}=\frac{\left(2 C_{2}\right)^{2}}{4 !}, \\
& C_{6}=\frac{C_{2} C_{4}}{15}=\frac{C_{2}^{3}}{90}=\frac{\left(2 C_{2}\right)^{3}}{720}=\frac{\left(2 C_{2}\right)^{3}}{6 !} \\
& C_{8}=\frac{2 C_{2} C_{6}+C_{4}^{2}}{2(63)}=\frac{\left(2 C_{2}\right)^{4}}{40,320}=\frac{\left(2 C_{2}\right)^{4}}{8 !} .
\end{aligned}
$$

This suggests the assumption that

$$
C_{2 j}=\frac{\left(2 C_{2}\right)^{j}}{(2 j) !}
$$

for all $j \geqslant 0$, since it is automatically true for $j=0$ and 1 , and has just been demonstrated to be true for $j=2,3$, and 4. To prove it inductively for all $j$, we assume that its truth has been demonstrated for all $j$ up to a certain value, say $j=N$, where $N$ is an integer equal to or greater than two. Then we use eq (22) to compute $C_{2 j}$ for $j=N+1$. Since all of the subscripts on the $C$ 's on the right side of eq (22) are less than or equal to $N$ we can use the assumption eq (23) for those $C$ 's with the result

$$
C_{2(N+1)}=\frac{\left(2 C_{2}\right)^{N+1}}{2\left(2^{2 N}-1\right)} \sum_{r=1}^{N} \frac{1}{(2 r) ![2(N-r+1)] !} .
$$

The sum on the right side of this equation can be evaluated by using the property of the binomial coefficients that (ref. [3])

$$
\sum_{r=0}^{N}\left(\begin{array}{l}
2 N \\
2 r
\end{array}\right)=2^{2 N-1} .
$$

From this property it follows by algebraic manipulation that

$$
\sum_{r=1}^{N} \frac{1}{(2 r) ![2(N-r+1)] !}=\frac{2\left(2^{2 N}-1\right)}{[2(N+1)] !} .
$$


Insertion of this value for the sum on the right side of eq (24) gives

$$
C_{2(N+1)}=\frac{\left(2 C_{2}\right)^{N+1}}{[2(N+1)] !}
$$

which is just eq (23) for $j=N+1$. This then furnishes the inductive step that allows us to assert the truth of eq. (23) for all $j$ from its demonstrated truth for $j \leqslant N$.

If we now set $2 C_{2}=K^{2}$ (where $K$ may be real or pure imaginary, depending on whether $C_{2}$ is positive or negative) and insert eq (23) for the coefficients into the series eq (14), we can recognize the resulting series as the Taylor expansion about $\alpha=0$ of $\cosh K \alpha$,

$$
h(\alpha)=\cosh K \alpha .
$$

Equation (27) is therefore the most general, regular solution of the "double angle cosine formula" eq (13). The lack of uniqueness of the solution is expressed by the arbitrariness of the parameter $K$.

From the definition eq (12) we have

$$
g(\alpha)=1+2 \cosh K \alpha .
$$

Since, from the first part of the periodicity property each element of $R(\alpha)$ has period $2 \pi$, so does $g(\alpha)$. This restricts $K$ to be the imaginary number $m i$ where $m$ is any positive or negative integer. The second part of the periodicity property, which requires that $g(\alpha)$ have no period smaller than $2 \pi$, selects the particular values $m= \pm 1$. The result for either value of $m$ is

$$
g(\alpha)=\operatorname{tr} R(\alpha)=1+2 \cos \alpha,
$$

which is eq (3).

\section{Solution of the Secular Equation}

The group property (6) allows us to replace $[R(\alpha)]^{3}$ and $[R(\alpha)]^{2}$ in eq (4) by $R(3 \alpha)$ and $R(2 \alpha)$. respectively. The resulting equation contains no rotation matrix raised to a power higher than the first.

$$
R(3 \alpha)-(1+2 \cos \alpha) R(2 \alpha)+(1+2 \cos \alpha) R(\alpha)-I=0 .
$$

We can therefore try to solve this equation by a Fourier series between 0 and $2 \pi$ in $\alpha$, rather than by a power series as was done with the trace equation. The advantage of such a Fourier series solution is that it automatically incorporates the periodicity property.

It turns out to be more practical for this problem to use a Fourier series for $R(\alpha)$ in the complex exponentials $e^{i m \alpha}$ rather than in $\sin m \alpha$ and $\cos m \alpha$. We write this series as

$$
R(\alpha)=\sum_{m=-\infty}^{\infty}\langle m \mid R\rangle e^{i m \alpha}
$$

In eq (30) we are using Dirac notation [4] for the Fourier coefficient matrices in order to avoid complicated expressions in subscripts, in equations for the coefficients to be derived soon. The Fourier coefficients are given by the usual formula

$$
\langle m \mid R\rangle=\frac{1}{2 \pi} \int_{0}^{2 \pi} R(\alpha) e^{-i m \alpha} d \alpha .
$$

The entire dependence of $R(\alpha)$ on the axis of rotation is contained in these Fourier coefficients.

We now substitute the series eq (30) evaluated for the appropriate arguments into each of the terms of eq (29). We also express the coefficients $1+2 \cos \alpha$ as $1+e^{i \alpha}+e^{-i \alpha}$. The resulting equation can be written

$$
\sum_{m=-\infty}^{\infty}\langle m \mid R\rangle\left[e^{3 i m \alpha}-e^{2 i m \alpha}+e^{i m \alpha}-e^{i(2 m+1) \alpha}+e^{i(m+1) \alpha}-e^{i(2 m-1) \alpha}+e^{i(m-1) \alpha}\right]=I .
$$


Equations for the Fourier coefficients are extracted from eq (32) by the use of the orthonormality property of the complex exponentials

$$
\int_{0}^{2 \pi} e^{i(r-k) \alpha} d \alpha=2 \pi \delta_{r k}
$$

where $\delta_{r k}$ is the Kronecker delta, equal to one if $r=k$ and equal to zero if $r \neq k$. Multiplication of eq (32) by $e^{-i k \alpha}$, and integration of the resulting equation with respect to $\alpha$ from 0 to $2 \pi$ yields, with the help of eq (33)

$$
\sum_{m=-\infty}^{\infty}\langle m \mid R\rangle\left[\delta_{k, 3 m}-\delta_{k, 2 m}+\delta_{k, m}-\delta_{k, 2 m+1}+\delta_{k, m+1}-\delta_{k, 2 m-1}+\delta_{k, m-1}\right]=\delta_{k 0} I .
$$

In eq (34) $k$ is any integer, positive, negative, or zero.

We divide the consideration of eq (34) into three separate cases based on the value of $k$.

(a) $k=0$.

For this case $\delta_{k 0}=1$. On the left side of eq (34), the first, second, and third terms contribute for $m=0$. The fifth term contributes for $m=-1$ while the seventh term contributes for $m=1$. The fourth and sixth terms do not contribute for any value of $m$. The equation then reduces to

$$
\langle 0 \mid R\rangle+\langle 1 \mid R\rangle+\langle-1 \mid R\rangle=I .
$$

(b) $k$ is a positive or negative even integer, but not zero.

We represent $k$ by $2 j$ where $j$ is any positive or negative integer. For this case $\delta_{k 0}=0$. On the left side of eq (34) the fourth and sixth terms do not contribute for any value of $m$. The relation between the coefficients for this case is

$$
\left\langle\frac{2}{3} j \mid R\right\rangle-\langle j \mid R\rangle+\langle 2 j \mid R\rangle+\langle 2 j-1 \mid R\rangle+\langle 2 j+1 \mid R\rangle=0 .
$$

(c) $k$ is a positive or negative odd integer.

We represent $k$ by $2 j+1$ where $j$ is a positive or negative integer, or zero. We again have $\delta_{k 0}=0$ in eq (34). On the left side of that equation the sole noncontributing term is the second one. The relation between the coefficients which follows from eq (34) for this case is

$$
\left\langle\frac{1}{3}(2 j+1) \mid R\right\rangle+\langle 2 j+1 \mid R\rangle-\langle j \mid R\rangle+\langle 2 j \mid R\rangle-\langle j+1 \mid R\rangle+\langle 2(j+1) \mid R\rangle=0 .
$$

Evaluation of the coefficients proceeds by setting $j$ equal to the first few of its allowed values in eqs (36) and (37), and trying to find a general trend which can then be tested inductively. In this process we set equal to zero any coefficient $\langle m \mid R\rangle$ in which $m$ is not an integer. Thus, setting $j=0$ in eq (37) and equating $\left\langle\frac{1}{3} \mid R\right\rangle$ to zero, we find that $\langle 2 \mid R\rangle=0$. Setting $j=1$ in eqs (36) and (37), leads to the results $\langle 3 \mid R\rangle=0,\langle 4 \mid R\rangle=0$. The value $j=-1$ leads to $\langle-2 \mid R\rangle=0,\langle-3 \mid R\rangle=0$. The value of $j=2$ leads to $\langle 5 \mid R\rangle=0,\langle 6 \mid R\rangle=0$, while $j=-2$ leads to $\langle-4 \mid R\rangle=0,\langle-5 \mid R\rangle=0$. The results $\langle 7 \mid R\rangle=\langle 8 \mid R\rangle=\langle-6 \mid R\rangle=\langle-7 \mid R\rangle=0$ follow from setting $j=3$ and then -3 .

These results suggest that $\langle m \mid R\rangle=0$ for all $|m| \geqslant 2$. To test this we follow the trend suggested by these results and assume it has been shown from eqs (36) and (37) for all integer values of $|j|$ up to and including some value $J$ greater than one, that

$$
\begin{gathered}
\langle 2 \mid R\rangle=\langle 3 \mid R\rangle=\ldots=\langle 2 J+1 \mid R\rangle=\langle 2(J+1) \mid R\rangle=0, \\
\langle-2 \mid R\rangle=\langle-3 \mid R\rangle=\ldots .=\langle-2 J \mid R\rangle=\langle-(2 J+1) \mid R\rangle=0 .
\end{gathered}
$$

We then set $|j|=J+1$ in eqs (36) and (37). This means that we have to consider the two values $j= \pm(J+1)$. For the plus sign eqs (36) and (37) yield, respectively

$$
\begin{gathered}
\left\langle\frac{2}{3}(J+1) \mid R\right\rangle-\langle J+1 \mid R\rangle+\langle 2(J+1) \mid R\rangle+\langle 2 J+1 \mid R\rangle+\langle 2 J+3 \mid R\rangle=0, \\
\left\langle\frac{1}{3}(2 J+3) \mid R\right\rangle+\langle 2 J+3 \mid R\rangle-\langle J+1 \mid R\rangle+\langle 2(J+1) \mid R\rangle-\langle J+2 \mid R\rangle+\langle 2(J+2) \mid R\rangle=0 .
\end{gathered}
$$

For $j=-(J+1)$ eqs (36) and (37) give, respectively

$$
\left\langle-\frac{2}{3}(J+1) \mid R\right\rangle-\langle-(J+1) \mid R\rangle+\langle-2(J+1) \mid R\rangle+\langle-(2 J+3) \mid R\rangle+\langle-(2 J+1) \mid R\rangle=0,
$$


$\left\langle-\frac{1}{3}(2 J+1) \mid R\right\rangle+\langle-(2 J+1) \mid R\rangle-\langle-(J+1) \mid R\rangle+\langle-2(J+1) \mid R\rangle-\langle-J \mid R\rangle+\langle-2 J \mid R\rangle=0$.

In eqs (39) and (40), the coefficients $\langle J+1 \mid R\rangle,\langle 2(J+1) \mid R\rangle,\langle 2 J+1 \mid R\rangle,\langle J+2 \mid R\rangle,\langle-(J+1) \mid R\rangle$, $\langle-(2 J+1) \mid R\rangle,\langle-J \mid R\rangle$, and $\langle-2 J \mid R\rangle$, vanish by the assumptions eqs (38) and the fact that $J>1$. The coefficients $\left\langle\frac{2}{3}(J+1) \mid R\right\rangle,\left\langle\frac{1}{3}(2 J+3) \mid R\right\rangle,\left\langle-\frac{2}{3}\left(J+1|R\rangle\right.\right.$, and $\left\langle-\frac{1}{3}(2 J+1) \mid R\right\rangle$ all vanish because in each case either the index is not an integer, or if an integer, the index falls in the range of eqs (38). After striking out the vanishing coefficients, the results of eqs (39) and (40) are

$$
\begin{gathered}
\langle 2 J+3 \mid R\rangle=\langle 2(J+1)+1 \mid R\rangle=0, \\
\langle 2(J+2) \mid R\rangle=\langle 2(J+1+1) \mid R\rangle=0, \\
\langle-2(J+1) \mid R\rangle=0, \\
\langle-(2 J+3) \mid R\rangle=\langle-[2(J+1)+1] \mid R\rangle=0 .
\end{gathered}
$$

These results extend the range of assumptions (38) from $|j|=J$ to $|j|=J+1$. We have therefore shown by induction that the only nonvanishing coefficients in the Fourier series eq $(30)$ are $\langle-1 \mid R\rangle$, $\langle 0 \mid R\rangle$, and $\langle 1 \mid R\rangle$.

The Fourier expansion of $R(\alpha)$ may now be written

$$
R(\alpha)=\langle 0 \mid R\rangle+\langle 1 \mid R\rangle e^{i \alpha}+\langle-1 \mid R\rangle e^{-i \alpha} .
$$

The coefficients in this expansion obey the restriction (35). We now define the matrix coefficients $L, M$, and $N$ from the equations

$$
\begin{aligned}
L & \equiv\langle 0 \mid R\rangle, \\
M & \equiv\langle 1 \mid R\rangle+\langle-1 \mid R\rangle, \\
N & \equiv-i(\langle 1 \mid R\rangle-\langle-1 \mid R\rangle) .
\end{aligned}
$$

The restriction (35) expressed in terms of $L, M$, and $N$, is

$$
L+M=I \text {. }
$$

The expansion (41) now takes the form

$$
R(\alpha)=I-M-N \sin \alpha+M \cos \alpha .
$$

In arriving at the form (44) we have assumed that $R(\alpha)$ obeys the group property. We now have to insure that it does by imposing the group property on eq (44). For this purpose it is sufficient to require the condition $[R(\alpha)]^{2}=R(2 \alpha)$. This then implies eq (6) for arbitrary $\alpha_{1}$ and $\alpha_{2}$. Equating $R(2 \alpha)$ to the square of the right side of eq (44), and expressing the quantities $\sin ^{2} \alpha, \cos ^{2} \alpha$, and $\sin \alpha \cos \alpha$ in terms of $\cos 2 \alpha$ or $\sin 2 \alpha$, we have

$I-M+M \cos 2 \alpha-N \sin 2 \alpha=2 M(I-M) \cos \alpha-(2 N-M N-N M) \sin \alpha-\frac{1}{2}(M N+N M) \sin 2 \alpha$

$$
+\frac{1}{2}\left(M^{2}+N^{2}\right)+(I-M)^{2}+\frac{1}{2}\left(M^{2}-N^{2}\right) \cos 2 \alpha .
$$

By equating the coefficients of similar Fourier components on the two sides of this equation, we arrive at the relations

$$
\begin{gathered}
M=-N^{2}, \\
N^{3}=-N .
\end{gathered}
$$

These relations show that all powers of $N$ higher than second are equivalent to either $N$ or $N^{2}$. Equation (44) may now be written

$$
R(\alpha)=I+N^{2}-N \sin \alpha-N^{2} \cos \alpha .
$$


It remains only to determine the matrix $N$. This is done by imposing both the first and second orthogonality conditions on the form (47). The most convenient way to impose the first orthogonality conditions is to consider an infinitesimal rotation. This leads to the restriction

$$
\widetilde{N}=-N \text {, }
$$

which states that $N$ is antisymmetric. Instead of imposing the complete second orthogonality conditions it is sufficient to impose a consequence of them, namely, the existence of an axis of rotation. Therefore if $n$ is a column matrix representing the axis of rotation, imposing the condition

$$
R(\alpha) n=n,
$$

places a restriction on $N$ which guarantees that eq (47) obeys the second orthogonality conditions. Equation (48) becomes

$$
N^{2} n-N^{2} n \cos \alpha-N n \sin \alpha=0,
$$

and the restriction which this places on $N$ is

$$
N n=0 .
$$

If the components of $n$ are formally regarded as known, the eqs (49) are three linear homogeneous equations for the determination of the three independent elements of $N$. These elements are linear functions of the components of $n$. The only antisymmetric matrix which can be formed linearly from $n$ is $\epsilon_{i j k} n_{j}$. Hence

$$
N_{i k}=a \epsilon_{i j k} n_{j}
$$

where $a$ is a constant. It can be determined by imposing the condition (46b) on eq (50). The result is that $a^{2}=1$. By choosing $a=+1$, we formally complete the identification of eq (47) with the solution (5).

The exponential representation (I 28) of $R(\alpha)$ is established by noting from eq (5) (or (47)) that

$$
\frac{d R}{d \alpha}=-N R
$$

Recognizing that $R(0)=I$, eq $(51)$ can be formally integrated to give

$$
R(\alpha)=e^{-N \alpha}
$$

To establish the equivalence between eqs (47) and (52), one expands eq (52) as a power series in $N \alpha$. By noting eq (46b), all powers of $N$ which arise are expressible as either $N$ or $N^{2}$. The resulting series is

$$
R(\alpha)=I-N\left(\alpha-\frac{\alpha^{3}}{3 !}+\frac{\alpha^{5}}{5 !}-\ldots\right)+N^{2}\left(\frac{\alpha^{2}}{2 !}-\frac{\alpha^{4}}{4 !}+\frac{\alpha^{6}}{6 !}-\ldots\right)
$$

The coefficient of $N$ can be recognized as the beginning of the series for $\sin \alpha$ while the coefficient of $N^{2}$ is seen to be the start of the series for $1-\cos \alpha$.

Note added in proof. Since the publication of papers I, II, and III, a reference [Gibbs, J. W., and Wilson, E. B., Vector Analysis, sec. 53. (Charles Scribner and Sons, New York, 1909; Dover Publications, Inc., New York, 1960)], has come to the author's attention in which the second orthogonality conditions are derived in a way which is similar to the method used in I, although not exhibited in matrix notation. Improper rotations are not taken into account. The relations which we have called the second orthogonality conditions were apparently known to mathematicians of the nineteenth century although no special name was given to them. The full exploration of the content of those equations, which has been the subject of this series of papers, does not appear to have been carried out until now. 
I thank Werner E. Sievers, John F. Sullivan, and William A. Whitcraft, Jr., for their support and encouragement during the course of this work.

\section{References}

[1] Gelman, H., The second orthogonality conditions in the theory of proper and improper rotations. I. Derivation of the conditions and of their main consequences, J. Res. NBS 72B (Math. Sci) No. 3, 229-237 (1968).

[2] Gelman, H., The second orthogonality conditions in the theory of proper and improper rotations. II. The intrinsic vector, J. Res. NBS 73B (Math. Sci.) No. 2, (1969).

[3] Beckenbach, E. F., ed., Applied Combinatorial Mathematics, sec. 3.2. (John Wiley \& Sons, Inc., New York, 1964).

[4] Dirac, P. A. M., The Principles of Quantum Mechanics 4th edition, chs. 1, 2, 3. (Oxford University Press, Oxford, 1958).

(Paper 73B2-301) 